\title{
Características Psicométricas e Estrutura Fatorial da FACES III numa Amostra de Famílias em Risco Psicossocial
}

\author{
Psychometric Characteristics and Factor Structure of FACES III in a Sample of \\ Families at Psychosocial Risk
}

\author{
Cristina Nunes ${ }^{1}$, Lara Ayala-Nunes ${ }^{2}$, Laura Inês Ferreira ${ }^{3}$ e Cátia Martins ${ }^{4}$
}

\begin{abstract}
Resumo
Este estudo visou a adaptação e exploração das características psicométricas da Escala de Avaliação da Coesão e Adaptabilidade Familiar (FACES III) com uma população de famílias portuguesas em risco psicossocial. Esta medida é a mais utilizada para a avaliação do funcionamento familiar de acordo com as dimensões do Modelo Circumplexo dos Sistemas Familiares. Participaram 388 pais de crianças com medidas de apoio e proteção, residentes no Algarve. A FACES III foi aplicada em conjunto com medidas de stresse parental, aliança parental e sentimento de competência parental para testar a validade de constructo. A análise fatorial exploratória confirmou a solução original de dois fatores. A Coesão demonstrou alta consciência interna e boa validade de constructo, podendo ser considerada uma medida fiável desta dimensão. A Adaptabilidade apresentou resultados insatisfatórios em todos os indicadores psicométricos, pelo que se aconselha a revisão desta subescala. Os resultados são discutidos considerando as diversas versões do instrumento.
\end{abstract}

Palavras-chave: adaptabilidade, análise fatorial, coesão, família, risco psicossocial

\begin{abstract}
This study aimed to adapt and explore the psychometric properties of the Family Adaptability and Cohesion Evaluation Scale (FACES III) with a sample of Portuguese families at psychosocial risk. This instrument is the most used for the evaluation of family functioning according to the dimensions of the Circumplex Model of Family Systems. The instrument was applied to 388 parents of children who were followed by the Child Protection Services in the Algarve (south of Portugal). The FACES III was applied along with measures of parenting stress, parenting alliance and parenting sense of competence to test construct validity. The exploratory factor analysis confirmed the original two-factor solution. Cohesion showed high internal consistency and good construct validity; thus, it can be considered as a satisfactory measure of this dimension. Adaptability, on the other hand, showed poor psychometric properties; therefore, we suggest that this subscale is revised to improve its shortcomings. Our main results are discussed considering the different versions of the instrument.
\end{abstract}

Keywords: adaptability, cohesion, factorial analysis, family, psychosocial risk

Este estudo foi financiado por uma bolsa sabática da Fundação para a Ciência e Tecnologia (SFRH/BSAB/114294/2016) e por fundos nacionais no âmbito do projeto CIP ( $\left.\operatorname{Ref}^{\mathrm{a}} \mathrm{UIDB} / \mathrm{PSI} / 04345 / 2020\right)$.

\footnotetext{
${ }^{1}$ Doutorada em Psicologia. Centro de Investigação em Psicologia (CIP). Universidade do Algarve, Campus de Gambelas, s/n. 8005-139 Faro, Portugal. Tel.: 919813927. E-mail: csnunes@ualg.pt

${ }^{2}$ Doutorada em Psicologia. Department of Psychiatry, University of Oxford. Warneford Hospital, Warneford Ln, Headington. Oxford OX3 7JX. United Kingdom. Tel.: 7745140703. E-mail: lara.ayala88@gmail.com

${ }^{3}$ Mestre em Psicologia Clínica e da Saúde. Universidade do Algarve, Campus de Gambelas, s/n. 8005-139 Faro, Portugal. Tel.: 918346808. E-mail: liferreira@ualg.pt

${ }^{4}$ Doutorada em Psicologia. Centro de Investigação em Psicologia (CIP). Universidade do Algarve, Campus de Gambelas, s/n. 8005-139 Faro, Portugal. Tel.: 912856351. E-mail: csmartins@ualg.pt

Revista Iberoamericana de Diagnóstico y Evaluación - e Avaliação Psicológica. RIDEP · No59 · Vol.2 · 49-61 · 2021

ISSN: 1135-3848 print /2183-6051online
} 


\section{Introdução}

As famílias em risco psicossocial vivem em circunstâncias sociais e relacionais que tornam difícil o adequado desempenho do papel parental. Nos países desenvolvidos, os sistemas de proteção da infância e as intervenções com as famílias de risco sofreram, nas últimas décadas do séc. $\mathrm{XX}$, mudanças muito substanciais. A mais relevante é a alteração de uma prática assistencial, centrada nas situações mais graves e que frequentemente separava os menores da sua família, para outra, de carácter mais preventivo, que promove $o$ fortalecimento e a preservação familiar (Rodrigo, Máiquez, Martín, \& Byrne, 2008).

Atualmente entende-se que existem certos níveis de risco que, embora sendo importantes, não atingem gravidade suficiente para que $o$ Estado separe o menor da família. Quando os progenitores vivem em circunstâncias pessoais e relacionais que tornam difícil o adequado desempenho do seu papel parental, a intervenção deverá, em primeiro lugar, contribuir para melhorar os recursos e fortalecer os fatores protetores do bem-estar do menor, deixando as medidas legais drásticas como último recurso (Rapsey \& Rolston, 2020).

Esta conceção sobre a intervenção familiar foi adotada pelo Conselho da Europa na Recomendação 19 acerca da política de apoio à parentalidade positiva (Committee of Ministers of the Council of Europe, 2006), apelando à criação e ativação de serviços de proteção da criança e da família, nomeadamente aquelas em risco, pelos Estados Membros. Além disso, a Recomendação 12 sobre os Direitos das Crianças e os Serviços Sociais postula que uma intervenção por parte destes serviços se deve basear na preservação familiar, especialmente perante famílias que enfrentam dificuldades (Committee of Ministers of the Council of Europe, 2011).

Uma condição necessária para a aplicação desta abordagem com sucesso é o conhecimento das necessidades específicas de cada família. Só a partir duma avaliação rigorosa da trajetória vital dos pais, das suas relações interpessoais e das suas circunstâncias sociais será possível compreender a dinâmica familiar (Hidalgo et al., 2018; Menéndez, Hidalgo, Lorence, \& Pérez, 2016; Menéndez, Jiménez, \& Hidalgo, 2011). Ora, uma avaliação rigorosa não é possível sem a utilização de instrumentos robustos e validados para esta população. Muitas das escalas disponíveis foram desenvolvidas a partir de estudos efetuados em populações normativas não portuguesas, carecendo de aferição para a população portuguesa e desconhecendo-se as suas propriedades psicométricas tanto na população comunitária como na população em risco psicossocial.

O estudo dos padrões de interação e do papel que desempenham os membros da família é importante para a identificação de aspetos disfuncionais que podem comprometer o bemestar familiar e dos menores (Howes, Cicchetti, Toth, \& Rogosch, 2000). O Modelo Circumplexo de Olson, Sprenkle e Russell (1979) é um dos mais compreensivos para o estudo dos sistemas familiares. Trata-se de um modelo sistémico, útil para o diagnóstico relacional da família, que descreve o funcionamento familiar a partir de três dimensões: a Coesão e a Adaptabilidade como fatores centrais e a Comunicação familiar, enquanto dimensão facilitadora.

A Coesão familiar refere-se aos laços familiares, ao apoio, à implicação familiar, aos limites ou fronteiras internas e externas, aos processos de tomada de decisão, à aliança entre pais e filhos e à autonomia. A Adaptabilidade diz respeito à estrutura de poder na família (liderança e disciplina), ao estilo de negociação, às funções e às regras de interação. A coesão familiar diz respeito ao sentimento de pertença e de apoio, revelando a intimidade emocional entre os seus membros, e a adaptabilidade ao poder e à influência dos familiares entre si (Olson \& Gorall, 2003).

A terceira dimensão é a Comunicação familiar, que diz respeito à capacidade de comunicação positiva existente entre o casal e/ou todos os membros do sistema familiar. A comunicação familiar envolve as habilidades de diálogo, escuta ativa, clareza, acompanhamento contínuo, respeito, consideração e empatia existentes entre os membros da família (Olson, 2011). De acordo com os autores, a Comunicação é vista não como uma dimensão separada mas como uma componente facilitadora da Coesão e Adaptabilidade, na medida em que os sistemas familiares com melhores níveis de comunicação 
apresentam igualmente melhores indicadores nas outras duas dimensões (Olson, Waldvogel, \& Schlief, 2019).

Segundo o Modelo Circumplexo, um funcionamento familiar saudável é descrito através de uma hipótese curvilínea, em que valores extremamente baixos ou extremamente elevados na Coesão e Adaptabilidade são representativos de um funcionamento disfuncional, com tendência a experimentar mais problemas nas suas relações e na adaptabilidade familiar ao longo do tempo. Como tal, um funcionamento adequado será descrito por níveis equilibrados das duas principais dimensões (Olson, 2011).

De acordo com os pressupostos do modelo relativos à Coesão, num funcionamento familiar saudável, os membros da família são independentes e simultaneamente unidos à sua família. Quanto à Adaptabilidade, um funcionamento saudável implica a capacidade para, em função das influências externas e mudanças evolutivas, adaptar-se de uma forma equilibrada e consistente, isto é, não comprometendo a identidade do sistema familiar e permitindo a manutenção da hierarquia da autoridade parental (Olson \& Gorall, 2003; Olson, Portner, \& Lavee, 1985; Olson et al., 2019).

No entanto, a solução bidimensional deste constructo não tem sido consistente nas diversas culturas nas quais foi testado. Por exemplo, Schmidt, Barreyro e Maglio (2009), numa amostra de famílias argentinas encontraram 3 fatores: coesão, flexibilidade 1 e flexibilidade 2. No México, Rosas, Clavelina, Trillo, Corja e Ibánez (2002) propuseram uma estrutura de quatro fatores, deparando-se com duas dimensões desconhecidas. Em todos os estudos, a dimensão de Adaptabilidade é aquela que tem sido objeto de maiores críticas quanto à sua validade. Concetualmente, esta dimensão poder-se-ia subdividir, por apresentar tanto conceitos relacionados com a flexibilidade e capacidade de adaptação como com a estrutura de liderança, regras e estilo de negociação existentes no sistema familiar (Jiménez, Lorence, Hidalgo, \& Menéndez, 2017; Schmidt, Barreyro, \& Maglio, 2009).

Para a avaliação das dimensões do funcionamento familiar têm sido utilizados diversos instrumentos, tais como a Family Assessment Device (FAD) (Epstein, Baldwin, \& Bishop, 1983, adaptada por Patrão, Pimenta, Água, \& Leal, 2020), a escala Family APGAR (Smilkstein, 1978, adaptada por Agostinho \& Rebelo, 1988), e a Escala de Avaliação da Adaptabilidade e Coesão Familiar III (Olson et al., 1985, adaptada por Curral et al., 1999).

As medidas mais utilizadas nos estudos que testam o Modelo Circumplexo (Olson et al., 2019) são o conjunto de Escalas de Avaliação da Adaptabilidade e Coesão Familiar, nas suas diferentes versões (FACES I, II, III e IV; Bell, 1980; Olson, 2011; Olson et al., 1985; Olson, Portner, \& Bell, 1982; Portner, 1981). A Escala de Avaliação da Adaptabilidade e Coesão Familiar III corresponde à terceira versão deste instrumento de autorrelato, e é composta por 20 itens, construídos originalmente para a avaliação operacional das dimensões de Coesão e Adaptabilidade dos sistemas familiares (Olson et al., 1985; Curral et al., 1999). Na FACES III, a dimensão de Coesão integra a avaliação de cinco subcategorias interrelacionadas, sendo elas o vínculo emocional, o apoio, os limites familiares, o lazer e amigos e os interesses. Da mesma forma, a escala de Adaptabilidade agrega quatro conceitos associados: liderança, controlo, disciplina, e as regras e papéis (Olson et al., 1985). Desta forma, a FACES III pode ser aplicada ao longo do todos os ciclos vitais da família e a todos os membros da família, a partir dos 12 anos de idade. Os estudos têm demonstrado que é um instrumento com bom poder discriminatório para diferenciar grupos familiares com e sem problemas clínicos (Forjaz, Cano, \& Cerevera-Enguix, 2002; Schmidt et al., 2009). Uma revisão sistemática das medidas de funcionamento familiar mais recentes (Hamilton \& Carr, 2016) destaca a FACES III como um instrumento de grande confiabilidade e utilização na literatura científica da área.

Não obstante o contributo científico da escala FACES III, alguns dos estudos de adaptação da medida têm vindo a encontrar dificuldades na replicação dos dados psicométricos da versão original (Jiménez et al., 2017). Bazo-Alvarez et al. (2016) confirmaram uma solução de dois fatores e concluíram que o FACES III tem fiabilidade e validez suficientes para ser utilizado com 
adolescentes peruanos numa avaliação grupal ou individual. Contudo, na Suíça, Vandeleur, Preisig, Fenton e Ferrero (1999) propuseram uma solução com 2 fatores, mas apenas após eliminar alguns itens. Também no Japão, num estudo com estudantes universitários, só foi possível obter uma estrutura de 2 fatores apresentando uma versão modificada da escala no que diz respeito à correspondência entre os itens e as dimensões (Hasui, Kishida, \& Kitamura, 2004). O mesmo se verificou na versão chilena, em que a primeira análise fatorial sugeria uma estrutura de sete fatores (Zegers, Larraín, Polaino-Lorente, Trapo, \& Diez, 2003).

Em Espanha, diferentes estudos encontraram diferentes soluções: Polaino-Lorente e Martínez (1995), num estudo com 545 casais com filhos, encontraram 6 fatores. Martínez-Pampliega, Iraurgi, Galíndez e Sanz (2006), com uma amostra de estudantes universitários, encontraram uma solução de dois fatores incluindo os 20 itens. Mas, num estudo posterior, os autores propõem outro modelo, com indicadores de ajustamento mais adequados e uma estrutura de 2 ordens de fatores: a $1^{\text {a }}$ ordem incluía as duas dimensões teóricas e a $2^{\mathrm{a}}$, uma dimensão global de funcionamento familiar (Martínez-Pampliega, Castillo, \& Vázquez, 2010).

Em Portugal, os estudos de validação da FACES III foram realizados com duas amostras clínicas (doentes com epilepsia e com esclerose múltipla) e um grupo de adultos da população geral (Curral et al, 1999). Os autores obtiveram valores de consistência interna de .80 para a dimensão coesão e .62 para a adaptabilidade. A partir de uma análise fatorial exploratória encontraram uma estrutura de seis fatores, sobretudo pela dispersão dos itens da subescala da adaptabilidade, que se repartem em 4 fatores.

De modo a ultrapassar alguns dos problemas identificados com a FACES III, foi criada uma nova versão, a FACES IV (Olson, 2011), constituída por 42 itens que se distribuem em seis subescalas, duas que permitem avaliar níveis equilibrados de funcionamento familiar (a Coesão e a Flexibilidade, nova designação de Adaptabilidade) e quatro subescalas que correspondem a composições desequilibradas (Desmembrada, Caótica, Rígida e Emaranhada). Esta medida veio acrescentar a possibilidade de avaliar distintas classificações do funcionamento familiar, além de as designar linearmente como funcionais ou disfuncionais (Olson, 2011).

No contexto português, Pereira e Teixeira (2013) conduziram uma investigação para validar a FACES IV, utilizando uma amostra de 214 adultos cuidadores de crianças em quimioterapia. Nesta validação, foram registados valores de consistência interna adequados, à exceção das subescalas Rígida e Emaranhada, que apresentavam baixa fiabilidade ( $\alpha$ de .67 para ambas as subescalas). Conclusões semelhantes foram encontradas por Silva e Sequeira (2015), em que a FACES IV foi aplicada a 387 famílias portuguesas, verificando-se que as subescalas Emaranhada e Rígida apresentaram níveis de consistência interna abaixo do desejável e baixa validade convergente.

Ainda em Portugal, estudos de validação da FACES IV mais recentes aplicaram o instrumento a adolescentes (Gouveia-Pereira, Gomes, Miranda, \& Candeias, 2020) e a adultos portugueses (Gomes, Peixoto, \& Gouveia-Pereira, 2019) e replicaram a estrutura original de seis fatores proposta pelo autor (Olson, 2011), com bons índices psicométricos, mas só após a retirada de alguns itens, o que resultou numa solução final de 24 itens (ao invés de 42). Nestas duas investigações, as subescalas Rígida e Emaranhada apresentam igualmente limitações, em termos de validade convergente e discriminante, pelo que os autores alertaram para a necessidade de maior exploração teórica destas dimensões (Gomes et al., 2019; Gouveia-Pereira et al., 2020).

Além destas limitações, a FACES IV é uma versão mais longa e, como tal, pode ser menos útil para protocolos de avaliação que pretendam integrar um maior número de medidas, de fácil e rápida aplicação. Por esse motivo, não obstante as desvantagens da FACES III, esta terceira versão continua a ser utilizada (e.g., Gouveia-Pereira, Abreu, \& Martins, 2014) e é considerada adequada para avaliar o funcionamento familiar, tanto pela sua fundamentação teórica, como pela sua aplicabilidade no âmbito clínico (AbreuAfonso \& Leal, 2016; Hamilton \& Carr, 2016). Contudo, poucos são os estudos de validação realizados com famílias em risco psicossocial. Alguns autores têm mostrado que as famílias em risco psicossocial apresentam pontuações mais 
baixas do que as da população geral, tanto ao nível da coesão como da adaptabilidade, e tendem a ser mais coesas do que adaptáveis (Hidalgo et al., 2009; Jiménez et al., 2017; Macedo, Nunes, Costa, Ayala-Nunes, \& Lemos, 2013; Pérez, Nunes, Nunes, \& Hidalgo, 2012; Sousa \& Ribeiro, 2005).

Importa destacar o estudo de validação da FACES III com 322 famílias em risco psicossocial das cidades de Sevilha e Huelva (Jiménez et al., 2017), no qual também não foi replicada a estrutura bidimensional da escala, indicando que apenas a dimensão da coesão (após a retirada de 3 itens) obtinha indicadores psicométricos aceitáveis.

Dada a relevância destas dimensões para a compreensão do funcionamento e ajustamento familiar e o facto de não existirem estudos de validação em Portugal com famílias em risco psicossocial, o presente estudo tem como objetivo adaptar e estudar as propriedades psicométricas das escalas de adaptabilidade e coesão familiar. Além disso, para avaliação da validade de construto, foram incluídas variáveis que apresentam uma associação teórica comprovada com as dimensões do funcionamento familiar, sendo elas o stresse parental, a aliança parental e a satisfação e eficácia parental. Deste modo, esperamos que as escalas de Coesão e Adaptabilidade apresentem uma associação negativa com o stresse parental e uma associação positiva com as restantes.

\section{Método}

\section{Participantes}

Foram entrevistados 388 pais, cujos filhos beneficiavam de uma medida de promoção e proteção nas Comissões de Proteção a Crianças e Jovens (CPCJ) do Algarve. Os participantes, na sua maioria eram mães (79.4\%; pais: $20.6 \%$ ), com idades compreendidas entre os 16 e os 75 anos $(M=38.19 ; \quad D P=9.15)$, com baixo nível de escolaridade (ensino básico incompleto $=48.5 \%$, ensino básico completo $=3.5 \%$, ensino secundário $=13.7 \%$, ensino superior $=4.4 \%$ ), que desempenhavam profissões com baixa qualificação $(70.1 \%$, nível médio $=25.1 \%$, nível elevado $=4.8 \%$ ). A maioria das famílias era biparental (68.7\%), com dois filhos em média $(D P=1.43)$, essencialmente do sexo masculino (62.4\%), cujas idades se encontravam entre os $0 \mathrm{e}$ os 18 anos $(M=10.95, D P=4.82)$.

Os participantes foram selecionados pelos técnicos das instituições utilizando os seguintes critérios: 1) ter pelo menos um filho menor de idade dependente; 2) sofrer vários problemas e situações de risco para os seus filhos que, embora importantes, não alcançassem um grau de severidade suficiente para justificar medidas de retirada.

\section{Instrumentos}

Escala de Avaliação da Adaptabilidade e Coesão Familiar (FACES III): utilizámos a tradução portuguesa de Curral et al. (1999) da Family Adaptability and Cohesion Evaluation Scale desenvolvida por Olson et al. (1985). Tal como descrito anteriormente, esta escala avalia duas dimensões do funcionamento familiar: a coesão (i.e., laços emocionais entre os membros da família) e a adaptabilidade (i.e., grau de flexibilidade da família para mudar regras e papéis, de modo a responder a problemas). É um instrumento de autopreenchimento, constituído por 20 itens, respondidos numa escala de cinco pontos (1=Nunca ou quase nunca; 2=Poucas vezes; $3=$ Às vezes; $4=$ Com frequência; ou 5=Quase sempre), onde os itens ímpares medem a Coesão Familiar (CF, 10 itens, e.g., "Os membros da nossa família pedem ajuda uns aos outros") e os pares avaliam a Adaptabilidade Familiar (AF, 10 itens, e.g., "Quando solucionamos problemas, costumamos ter em conta as opiniões dos nossos filhos"). Pontuações mais elevadas correspondem a níveis mais elevados de coesão e adaptabilidade. A pilotagem desta versão permitiu identificar alguns problemas de compreensão nos participantes, pelo que as instruções foram simplificadas e a redação de alguns itens foi adaptada para garantir a acomodação do instrumento ao nível cultural e educativo dos participantes (Carretero-Dios \& Pérez, 2005). No presente estudos obtiveram-se os seguintes índices de fiabilidade: $\alpha=.79$ para Coesão e $\alpha=.52$ para Adaptabilidade.

Competência Parental Percebida (PSOC): esta escala, originalmente desenvolvida por GibaudWallston e Wandersman (1978) e posteriormente 
adaptada por Johnston e Mash (1989), avalia a competência percebida como pai ou mãe através de duas dimensões: a eficácia e a satisfação. A dimensão Eficácia (EP) é composta por sete itens que avaliam em que medida o progenitor se sente competente nesse papel (ex.: "Apesar de ser difícil, eu já sei como se pode influenciar os filhos"). Por sua vez, a dimensão Satisfação (SP) possui nove itens e pretende determinar o grau em que o pai ou a mãe se sente satisfeito com o seu papel parental (ex.: "Com a idade que o meu filho tem, ser mãe não é agradável"). No total, o questionário é composto por 16 itens com seis respostas possíveis, que oscilam entre o 1 (não, discordo totalmente) e o 6 (sim, concordo totalmente). As pontuações mínimas e máximas possíveis para a dimensão Eficácia são 7 e 42, respetivamente; e para a dimensão Satisfação podem oscilar entre 9 e 54 . Pontuações superiores correspondem a uma perceção mais elevada de eficácia e satisfação parentais. No presente estudo, utilizámos a versão portuguesa de Nunes, Jiménez, Menéndez, Ayala-Nunes, \& Hidalgo (2016) e obtiveram-se os seguintes índices de fiabilidade: $\alpha=.75$ para eficácia e $\alpha=.70$ para satisfação parental.

Parenting Stress Index - Short Form (PSI-SF): esta escala, desenvolvida por Abidin (1990), tem uma versão adaptada para o Português (Santos, 2008), que foi usada no presente trabalho. Avalia três domínios do stresse associado ao papel parental com crianças até os 12 anos: o mal-estar parental, a interação disfuncional entre o pai e a criança, e o grau em que o pai percebe o seu filho como sendo uma criança difícil. Os itens têm cinco possibilidades de resposta (1=discordo totalmente a $5=$ concordo totalmente). O Mal-estar Parental (MP) inclui 12 itens que pretendem quantificar os sentimentos negativos experienciados pelo pai no seu papel de cuidador (ex.: "Sinto-me limitado por causa das minhas responsabilidades como pai"). Por sua vez, a subescala Interação Disfuncional ProgenitorCriança (IDPC; 12 itens) foca-se na perceção do progenitor de que o seu filho não cumpre as suas expectativas, as interações que mantêm não são positivas e não se sente aceite nem vinculado ao seu filho (ex.: "Quando faço coisas pelo meu filho tenho a sensação que o meu esforço não é muito apreciado"). A subescala Criança Difícil (CD; 12 itens) avalia as características comportamentais da criança que fazem com que seja fácil ou difícil lidar com ela (aceitação, humor e adaptabilidade). As pontuações mínimas e máximas para cada dimensão do instrumento são 12 e 60 , respetivamente. Para a escala total, as pontuações podem oscilar entre 36 e 180. Quanto mais elevada for a pontuação da escala, maior é o nível de stresse parental. No presente estudos obtiveram-se os seguintes índices de fiabilidade: $\alpha=.82$ para mal-estar parental, $\alpha=.74$ para Interação disfuncional e $\alpha=.85$ para Criança difícil.

Aliança Parental (PAI): Utilizámos a versão portuguesa (Ayala-Nunes, Nunes, \& Lemos, 2013) do Parental Alliance Inventory (Abidin \& Bruner, 1995). Este instrumento é composto por 20 itens que, numa escala de 1 a 5 , avaliam a relação de apoio e confiança que existe entre o casal enquanto pais (ex.: "Faço tudo que é possivel para falar com o meu marido sobre os nossos filhos"). No presente estudo obteve-se o seguinte índice de fiabilidade: $\alpha=.96$.

\section{Procedimentos}

Após o estabelecimento de protocolos de colaboração com as CPCJs do Algarve, que intervêm junto destas famílias, os técnicos das instituições colaboradoras selecionaram os progenitores que cumpriam os critérios de inclusão descritos anteriormente e convidaramnos a participar no estudo. Posteriormente, os entrevistadores, que tinham recebido formação, deslocaram-se às instituições para aplicar os instrumentos. Quando os participantes mostravam dificuldades de leitura os instrumentos foram aplicados através de entrevista individual.

Antes de proceder à aplicação dos questionários e à assinatura do consentimento informado, os participantes foram informados acerca dos objetivos do estudo, do carácter não compensatório da sua participação, da natureza anónima e confidencial das suas respostas e da possibilidade de desistir do estudo em qualquer momento sem que isso representasse quaisquer consequências para eles.

\section{Análises estatísticas}

A codificação e tratamento estatístico dos dados foram realizados com o IBM SPSS 25 e o 
programa FACTOR 9.2 (Lorenzo-Seva \& Ferrando, 2006). Em primeiro lugar foi examinada a existência de casos extremos multivariantes através do cálculo da distância de Mahalanobis (Tabachnick \& Fidell, 2019) e foram calculadas as estatísticas descritivas das escalas originais. Seguidamente foi analisada a normalidade na distribuição univariante dos itens, tendo em conta os índices de assimetria e curtose (i.e., valores compreendidos entre os intervalos \pm 1 e \pm 2 , respetivamente) tal como recomendam Ferrando e Anguiano-Carrasco (2010). Após este procedimento foi analisada a capacidade discriminante de cada item através do coeficiente de correlação corrigido entre a pontuação do item e o total da escala (>.25) (Nunnally \& Berstein, 1994) e a fiabilidade (i.e., caso o item obtivesse uma pontuação inferior ao da dimensão global, seria eliminado (Hair, Anderson, Tatham, \& Black, 2009).

Dado que a solução bidimensional da escala não tem sido validada de forma idêntica nas diversas culturas nas quais tem sido testada, optámos por analisar a dimensionalidade do instrumento através de uma análise fatorial exploratória com o programa FACTOR Vs. 9.2 (Lorenzo-Seva \& Ferrando, 2006). Foram comprovados os pressupostos de normalidade e linearidade entre cada par de variáveis. Tendo em conta que se tratava de uma escala ordinal, trabalhámos com a matriz de correlações policóricas e foi estimada a fiabilidade através o coeficiente de alfa ordinal. Como método de estimação utilizámos os mínimos quadrados não ponderados e uma rotação oblíqua, mediante o método Oblimin direto normalizado. A fatorabilidade da matriz foi estabelecida através de valores elevados na medida de adequação da amostra de Kaiser-Meyer-Olkin e de um resultado significativo no teste de esfericidade de Barlett (Carretero-Dios \& Pérez, 2005; Tabachnick \& Fidell, 2019).

Para decidir o número de fatores a reter foram considerados os seguintes critérios: valores próprios $\geq 1$, mínimo de três variáveis em cada fator (Ferrando \& Anguiano-Carrasco, 2010), análise paralelo com a implementação ótima de Timmerman e Lorenzo-Seva (2011), interpretabilidade e pertinência teórica. Foram retidos os itens com um coeficiente de configuração superior a .45 unicamente num dos fatores. Para avaliar a bondade do ajuste da solução fatorial foram analisados os índices de simplicidade S e LS (próximos a 1), assim como os índices GFI (recomendável > .95) e RMSR (próximo a 0) (Ferrando \& Anguiano-Carrasco, 2010; Hair et al., 2009).

A consistência interna dos fatores obtidos foi avaliada através do cálculo dos índices do alfa ordinal e das estatísticas descritivas. A validade de critério da versão proposta foi analisada através dos índices de correlação de Pearson dos fatores obtidos com as pontuações nas dimensões de aliança parental, competências parentais percebidas e stresse parental.

\section{Resultados}

\section{Análise descritiva inicial}

Foram identificados 4 casos (1\%) de extremos multivariados através do cálculo da distância de Mahalanobis, que foram eliminados das análises posteriores $(N=384)$. De acordo com a versão original dos autores, as escalas apresentaram os seguintes valores (Quadro 1).

Quadro 1. Descritivos da FACES III

\begin{tabular}{lcc} 
& Adaptabilidade & Coesão \\
\hline Média & 27.87 & 38.09 \\
Desvio-padrão & 5.23 & 6.36 \\
Mediana & 28.00 & 39.00 \\
Moda & 30.00 & 40.00 \\
Mínimo - Máximo & $16-43$ & $20-50$ \\
Assimetria & 0.21 & -0.41 \\
Curtose & -0.25 & -0.21 \\
$\alpha$ & .52 & .79 \\
\hline
\end{tabular}

Ambas dimensões se correlacionaram entre si significativa e positivamente $(r=.24 ; p<.000)$ e as pontuações foram mais elevadas na coesão do que na adaptabilidade $(t(383)=27.73 ; p<.001)$. Em seguida procedeu-se à análise fatorial exploratória.

\section{Estrutura fatorial}

Devido aos elevados índices de assimetria e curtose, foram eliminados das análises posteriores os itens 12, 18, 19 e 20 (Quadro 2). Os itens 6 e 16 não obtiveram correlações com o resto da escala $(r>.25)$, pelo que não foram utilizados nas análises seguintes. Por outro, lado a eliminação do 
Quadro 2. Análise da capacidade discriminante dos 20 itens originais da FACES III (N=186)

\begin{tabular}{lcccccc}
\hline & $M$ & $D P$ & Assimetria & Curtose & $\begin{array}{c}r \text { item-total } \\
\text { corrigida }\end{array}$ & $\begin{array}{c}\alpha \text { se eliminar o } \\
\text { item }\end{array}$ \\
\hline Item 1 & 3.54 & 0.98 & 0.03 & -0.72 & .33 & .76 \\
Item 3 & 3.67 & 1.15 & -0.42 & -0.72 & .30 & .77 \\
Item 5 & 3.75 & 1.06 & -0.63 & -0.06 & .45 & .74 \\
Item 7 & 3.81 & 1.20 & -0.81 & -0.26 & .34 & .76 \\
Item 9 & 3.88 & 1.00 & -0.61 & -0.25 & .54 & .73 \\
Item 11 & 3.97 & 1.05 & -0.70 & -0.38 & .63 & .71 \\
Item 13 & 3.79 & 1.17 & -0.61 & -0.55 & .47 & .74 \\
Item 15 & 3.58 & 1.13 & -0.40 & -0.79 & .63 & .71 \\
Item 17 & 3.47 & 1.24 & -0.38 & -0.81 & .37 & .76 \\
Item 19 & 4.66 & 0.67 & -2.26 & 6.04 & - & - \\
& & & & & & .76 \\
Item 2 & 3.11 & 1.27 & -0.32 & -0.79 & .39 & .51 \\
Item 4 & 2.93 & 1.34 & 0.02 & -1.07 & .35 & .52 \\
Item 6 & 2.18 & 1.36 & 0.80 & -0.73 & .10 & .61 \\
Item 8 & 2.77 & 1.11 & -0.03 & -0.75 & .41 & .50 \\
Item 10 & 2.08 & 1.25 & 0.79 & -0.57 & .35 & - \\
Item 12 & 1.27 & 0.66 & 2.80 & 8.55 & - & .54 \\
Item 14 & 2.56 & 1.14 & 0.29 & -0.53 & .31 & .57 \\
Item 16 & 2.54 & 1.48 & 0.42 & -1.29 & .22 & - \\
Item 18 & 4.16 & 1.11 & -1.38 & 1.17 & - & - \\
Item 20 & 4.19 & 1.04 & -1.15 & 0.58 & - & .58 \\
\hline
\end{tabular}

Quadro 3. Resultados da ACP com fins confirmatórios da FACES III

\begin{tabular}{lcccc}
\hline & \multicolumn{2}{c}{ Matriz padrão } & \multicolumn{2}{c}{ Matriz de estrutura } \\
\cline { 2 - 5 } & F1 & F2 & F1 & F2 \\
\hline Item 1 & .47 & .01 & .47 & .05 \\
Item 2 & .12 & .63 & .18 & .65 \\
Item 3 & .42 & -.01 & .42 & .03 \\
Item 4 & -.04 & .67 & .02 & .67 \\
Item 5 & .59 & .06 & .60 & .12 \\
Item 7 & .51 & -.17 & .50 & -.12 \\
Item 8 & -.16 & .70 & -.09 & .68 \\
Item 9 & .67 & .01 & .67 & .07 \\
Item 10 & -.02 & .55 & .03 & .55 \\
Item 11 & .77 & -.09 & .76 & -.01 \\
Item 13 & .64 & -.11 & .63 & -.05 \\
Item 14 & -.11 & .62 & -.04 & .61 \\
Item 15 & .75 & .08 & .75 & .15 \\
Item 17 & .46 & .46 & .50 & .50 \\
\hline
\end{tabular}

item 6 melhorava a fiabilidade da dimensão adaptabilidade. Por estes motivos, nas análises posteriores não foram incluídos os itens $6,12,16$, 18,19 e 20.

A fatorabilidade da matriz foi calculada, tendo obtido um valor aceitável na prova de KaiserMeyer-Olkin $(\mathrm{KMO}=.73)$ e um valor significativo no teste de esfericidade de Bartlett $\left(\chi^{2}(91)=522.50 ; \quad p<.001\right) . \quad$ A análise fatorial ofereceu uma solução de dois fatores com valores próprios $>1$, que contribuiu a explicar uma variância de $49.5 \%$ e os resultados da Análise Paralela recomendaram reter ambos fatores. $\mathrm{O}$ primeiro fator explicava $30 \%(\lambda=3.63)$, incluía os itens $1,3,5,7,9,11,13,15$ e 17 , reproduzindo quase na totalidade a dimensão original de coesão, isto é, os laços emocionais entre os membros da família. O segundo fator, que se refere à capacidade de adaptação da família, explicou uma variância de $19.5 \%(\lambda=2.35)$ e apenas reteve os itens $2,4,8,10$ e 14 da escala original de adaptabilidade.

Os indicadores de bondade de ajuste para esta solução foram satisfatórios (GFI=.97; RMSR=.06; $\mathrm{S}=.99 ; \mathrm{LS}=.64$ ).

Dado que o item 17 saturava nos dois fatores com valores $(r \geq .40)$, foi eliminado das análises posteriores (Quadro 3). Os valores de ajustamento obtidos após a eliminação do item 17 mantiveramse aceitáveis, GFI=.97; RMSR=.06; S=.99; $\mathrm{LS}=.68$, observando-se um ligeiro incremento no LS.

A análise descritiva de ambos fatores mostra uma média de $29.99(D P=5.31)$ para a coesão e $13.46 \quad(D P=3.94)$ para adaptabilidade. Os coeficientes de fiabilidade foram de um alfa ordinal de .76 e .65 respetivamente. Ambos fatores não se relacionam significativamente $(r=.01 ; p=.897)$.

\section{Validade de constructo}

Como podemos observar na Quadro 4, existe uma relação significativa entre a coesão familiar e as dimensões das demais variáveis, sendo esta negativa no que se refere ao stresse parental (i.e., mal-estar parental, interação disfuncional progenitor-criança e criança difícil) e positiva com a eficácia, satisfação e aliança parental. No entanto, a dimensão adaptabilidade não se relacionou com nenhuma destas dimensões. 
Quadro 4. Relações entre a Coesão, Adaptabilidade e outras dimensões em estudo

\begin{tabular}{lcccccc}
\hline & PSI-MP & PSI-IDPC & PSI-CD & PSOC-EP & PSOC-SP & PAI \\
\hline Coesão & $-.38 * * *$ & $-.34 * * *$ & $-.38 * * *$ & $.34 * * *$ & $.40 * * *$ & $.41 * * *$ \\
Adaptabilidade & .05 & .08 & .03 & -.02 & -.01 & .06 \\
\hline Nota. $* p<.05 ; * * p<.01 ; * * * p<.001$ & & & & &
\end{tabular}

\section{Discussão}

O objetivo principal do presente estudo foi adaptar a Escala de Avaliação da Adaptabilidade e Coesão Familiar III (FACES III; Olson et al., 1985) para famílias portuguesas em risco psicossocial, permitindo conhecer qual a estrutura fatorial do instrumento quando aplicado a uma amostra com caraterísticas clínicas.

Os resultados replicaram algumas das características psicométricas do estudo original e de outras investigações que se realizaram com populações em risco (e.g., Jiménez et al., 2017; Vandeleur et al., 1999). A subescala de Coesão da FACES III reteve a maioria dos itens da escala original ( 8 de 10) com um índice de fiabilidade razoável. Obtivemos igualmente evidências de validez de critério através da sua relação com outras dimensões relevantes do funcionamento e bem-estar familiar (aliança parental, stresse parental, eficácia e satisfação parental). Podemos concluir que esta subescala é um instrumento útil para a avaliação das forças e fragilidades das famílias em risco psicossocial.

Contrariamente, a escala de Adaptabilidade apresentou uma fiabilidade fraca, reteve poucos itens da escala original (5 de 10) e não se relacionou com nenhuma das dimensões utilizadas para a avaliação da validade de constructo. Os problemas relativos a esta subescala são congruentes com as conclusões apresentadas por outras investigações, nacionais e internacionais, em que a Adaptabilidade apresentou valores insatisfatórios (Curral et al., 1999; Hasui et al., 2004; Rolim, Lopes, Rodrigues, \& Coelho, 2006; Zegers et al., 2003).

Os problemas com esta subescala podem dever-se a vários aspetos. Um dos principais motivos apontados para justificar os valores alfa baixos está relacionado com a complexidade e multiplicidade de fatores associados à adaptabilidade, sugerindo-se que os itens construídos para esta dimensão podem refletir diferentes facetas da mesma (Jiménez et al., 2017;
Schmidt et al., 2009; Vielva, Pantoja, \& Abeijón, 2001). Por essa razão, alguns autores propõem uma solução de 3 fatores, na qual, por exemplo (Schmidt et al., 2009), a escala original de Adaptabilidade é dividida em dois: Flexibilidade 1 e Flexibilidade 2. Analisando comparativamente o conteúdo dos itens mantidos e dos retirados do Fator 2 do presente estudo, podemos encontrar disparidades. Os itens que saturaram no Fator da Adaptabilidade são itens que contêm um teor de adaptação à mudança e consideração por todos os membros da família (e.g., Item 2 - "Quando solucionamos problemas, costumamos ter em conta as opiniões dos nossos filhos"; Item 8 - "Na nossa família mudamos a maneira de fazer as coisas"), enquanto que os itens retirados parecem corresponder a questões de liderança, regras e papéis no sistema familiar (e.g., Item 6 - "Há distintas pessoas que mandam dentro da nossa família"; Item 16 - "Fazemos turnos para as responsabilidades da casa”).

Alguns destes estudos indicam a necessidade de se realizar uma distinção mais clara entre os subtópicos inerentes ao constructo de Adaptabilidade, que podem estar a captar diferentes aspetos desse mesmo constructo (Crowley, 1998; Schmidt et al., 2009). Para além disso, outros investigadores também têm salientado que os valores psicométricos verificados na subescala de Adaptabilidade se devem à formulação dos itens e à construção do instrumento (Jiménez et al., 2017). A avaliação funcional da adaptabilidade não pode apenas medir se a família tem ou não capacidade para mudar e adaptar-se. Deve tentar medir qual a direção e magnitude dessa mudança para poder determinar se essa capacidade é saudável e funcional (Schmidt et al., 2009). Em populações de risco, torna-se ainda mais pertinente procurar conhecer quais esses critérios, uma vez que pontuações extremadas podem ser mais frequentes e servirem como reflexo do conteúdo de adversidade do sistema familiar (Menéndez et al., 2011; Jiménez et al., 2017). 
Estes resultados sugerem que a subescala de Adaptabilidade deve ser repensada (MartínezPampliega et al., 2006) e que a medida FACES III pode vir a necessitar de alterações estruturais para que possa ser utilizada em contextos familiares de risco psicossocial. As alterações introduzidas na mais recente versão do instrumento - FACES IV (Gomes et al., 2019; Gouveia et al., 2020; Olson, 2011; Silva \& Sequeira, 2015) parecem ultrapassar alguns dos problemas encontrados, nomeadamente no que diz respeito à dimensão da adaptabilidade, denominada flexibilidade familiar nesta nova versão.

Como proposta de estudos futuros, propõe-se a realização de estudos psicométricos utilizando a versão mais atual do instrumento, a FACES IV, analisando a sua adequação às famílias em risco psicossocial. Por outro lado, seria também útil dispor de uma amostra mais diversa e analisar a variância entre diferentes grupos (por exemplo, famílias com e sem risco psicossocial, bi e monoparentais, pais e mães) e que se recorra a outros métodos de análise de confiabilidade como o teste-reteste e a medidas de validade convergente e discriminante do instrumento.

\section{Conclusão}

O presente estudo oferece conclusões importantes acerca das propriedades psicométricas da FACES III numa população de famílias em risco psicossocial. Os resultados demonstram que é um instrumento de fácil aplicação e útil para a avaliação do funcionamento familiar desta amostra, particularmente no que concerne à subescala de Coesão Familiar. A fiabilidade dessa dimensão tem sido sistematicamente comprovada, pelo que a sua interpretação é confiável.

Ainda assim, a investigação acerca das caraterísticas psicométricas do instrumento parece não estar terminada, nomeadamente no que diz respeito à subescala de Adaptabilidade. Como tal, é necessário continuar a exploração de melhores soluções para a avaliação das diferentes facetas abordadas pelo Modelo Circumplexo.

\section{Referências}

Abidin, R. R. (1990). Parenting Stress Index (3rd ed.). Charlottesville, VA: Pediatric Psychology Press.

Abidin, R. R., \& Brunner, J. F. (1995). Development of a parenting alliance inventory. Journal of Clinical Child Psychology, 24(1), 31-40. doi:10.1207/s15374424jccp2401_4

Abreu-Afonso, J., \& Leal, I. (2016). FACES III. Adaptação e validação da Family Adaptability and Cohesion Evaluation Scale (FACES), de H. Olson, J. Portner e Y Lavee, versão de Casal, para a população portuguesa. Mosaico, 63, 92-107.

Agostinho, M., \& Rebelo, L. (1988). Família: Do conceito aos meios de comunicação. Revista Portuguesa de Saúde Pública, 5(32), 18-21.

Ayala-Nunes, L., Nunes, C., \& Lemos, I. (2013). Parenting alliance among mothers of psychosocially at-risk families. Spatial and Organizational Dynamics Discussion Papers, 13, 18-30.

https://ideas.repec.org/p/ris/cieodp/2014_002. html

Bazo-Alvarez, J. C., Bazo-Alvarez, O. A., Aguila, J., Peralta, F., Mormontoy, W., \& Bennett, I. M. (2016). Propiedades psicométricas de la escala de funcionalidad familiar FACES-III: Un estudio en adolescentes peruanos. Revista Peruana de Medicina Experimental y Salud Pública, 33(3), 462-470. doi:10.17843/rpmesp.2016.333.2299

Bell, R. Q. (1980). Parent/adolescent relationships in families with runaways: Interaction types and circumplex model. Unpublished doctoral thesis, University of Minnesota.

Carretero-Dios, H., \& Pérez, C. (2005). Normas para el desarrollo y revisión de estudios instrumentales. International Journal of Clinical and Health Psychology, 5(3), 521551. Acedido em http://aepc.es/ijchp/NDREI07_es.pdf

Committee of Ministers of the Council of Europe (2006). Recommendation 19. Acedido em https://wcd.coe.int/View-Doc.jsp?id=10 73 507 
Committee of Ministers of the Council of Europe (2011). Recommendation 12. Acedido em https://wcd.coe.int/ViewDoc. $\quad$ jsp?id=187 2121

Crowley, S. L. (1998). A psychometric investigation of the FACES-III: Confirmatory factor analysis with replication. Early Education \& Development, 9(2), 161-178. doi:10.1207/s15566935eed0902_4

Curral, R., Dourado, F., Roma-Torres, A., Barros, H., Palha, A., \& Almeida, L. (1999). Coesão e adaptabilidade familiares numa amostra portuguesa: Estudo com o FACES III. Psiquiatría Clínica, 20(3), 213-217.

Epstein, N. B., Baldwin, L. M., \& Bishop, D. S. (1983). The McMaster Family Assessment Device. Journal of Marital and Family Therapy, 9(2), 171-180.

doi:10.1111/j.1752-0606.1983.tb01497.x

Ferrando, P. J., \& Anguiano-Carrasco, C. (2010). El análisis factorial como técnica de investigación en psicología. Papeles del Psicólogo, 31(1), 18-33. Acedido em http://www.papelesdelpsicologo.es/pdf/1793.p df

Forjaz, M. J., Cano, P. M., \& Cervera-Enguix, S. (2002). Confirmatory factor analysis, reliability and validity of a Spanish version of FACES III. American Journal of Family Therapy, 30(5), 439-449. doi:10.1080/01926180260296332

Gibaud-Wallston, J., \& Wandersman, L. P. (1978). Parenting sense of competence scale. New Jersey, EUA: Lawrence Erlbaum Associates.

Gomes, H. M. S., Peixoto, F., \& Gouveia-Pereira, M. (2019). Portuguese validation of the family adaptability and cohesion evaluation scaleFACES IV. Journal of Family Studies, 25(4), 477-494. doi:10.1080/13229400.2017.1386121

Gouveia-Pereira, M., Abreu, S., \& Martins, C. (2014). How do families of adolescents with suicidal ideation behave? Psicologia: Reflexão e Critica, 27(1), 171-178. doi:10.1590/S0102-79722014000100019

Gouveia-Pereira, M., Gomes, H., Miranda, M., \& Candeias, M. D. J. (2020). Coesão e flexibilidade familiar: Validação do pacote
FACES IV junto de adolescentes portugueses. Análise Psicológica, 38(1), 111-126.

doi:10.14417/ap.1651

Hamilton, E., \& Carr, A. (2016). Systematic review of self-report family assessment measures. Family Process, 55(1), 16-30. doi:10.1111/famp. 12200

Hair, J. F., Anderson, R. E., Tatham, R. L., \& Black, W. C. (2009). Multivariate data analysis ( $7^{\text {th }}$ ed.). New York: Prentice Hall.

Hasui, C., Kishida, Y., \& Kitamura, T. (2004). Factor structure of the FACES-III in Japanese university students. Family Process, 43(1), 133-140. doi:10.1111/j.1545-5300.2004.04301010.x

Hidalgo, M. V., Lorence, L., Pérez, J., Ménendez, S., Sánchez, J., Jiménez, L., \& Arenas, A. (2009). El apoyo social de mujeres solas con responsabilidad familiar. Espanha: Instituto Andaluz de la Mujer.

Hidalgo, V., Pérez-Padilla, J., Sánchez, J., AyalaNunes, L., Maya, J., Grimaldi, V., \& Menéndez, S. (2018). An analysis of different resources and programmes supporting at-risk families in Spain. Early Child Development and Care, 188(11), 1527-1538. doi:10.1080/03004430.2018.1491560

Howes, P. W., Cicchetti, D., Toth, S. L., \& Rogosch, F. A. (2000). Affective, organizational, and relational characteristics of maltreating families: A system's perspective. Journal of Family Psychology, 14(1), 95-110. doi:10.1037//0893-3200.14.1.95

Jiménez, L., Lorence, B., Hidalgo, V., \& Menéndez, S. (2017). Análisis factorial de las escalas FACES (Family Adaptability and Cohesion Evaluation Scales) con familias en situación de riesgo psicosocial. Universitas Psychologica, 16(2), 140-151.

doi:10.11144/Javeriana.upsy16-2.afef

Johnston, C., \& Mash, E. J. (1989). A measure of parenting satisfaction and efficacy. Journal of Clinical and Child Psychology, 18(2), 167175. doi:10.1207/s15374424jccp1802_8

Lorenzo-Seva, U., \& Ferrando, P. J. (2006). FACTOR. A computer program to fit the exploratory factor analysis model. Behavior Research Methods, 38(1), 88-91. doi:10.3758/BF03192753 
Macedo, C., Nunes, C., Costa, D., Ayala-Nunes, L., \& Lemos, I. (2013). Apoio social, acontecimentos stressantes, adaptabilidade e coesão em famílias em risco psicossocial. Psicologia, Saúde \& Doenças, 14(2), 304312.

Martínez-Pampliega, A., Castillo, I. I., \& Vázquez, M. S. (2010). Validez estructural del FACES-20Esp: Versión española de 20 ítems de la Escala de Evaluación de la Cohesión y Adaptabilidad Familiar. Revista Iberoamericana de Diagnóstico y Evaluación - e Avaliação Psicológica, 1(29), 147-165. Recuperado de https://www.aidep.org/03_ridep/R29/r29art8.p df

Martínez-Pampliega, A., Iraurgi, I., Galíndez, E., \& Sanz, M. (2006). Family Adaptability and Cohesion Evaluation Scale (FACES): Desarrollo de una versión de 20 ítems en español. International Journal of Clinical and Health Psychology, 6(2), 317-338. Recuperado de http://www.aepc.es/ijchp/articulos_pdf/ijchp180.pdf

Menéndez, S., Jiménez, L., \& Hidalgo, M. V. (2011). Estructura factorial de la escala PSOC (Parental Sense of Competence) en una muestra de madres usuarias de servicios de preservación familiar. Revista Iberoamericana de Diagnóstico y Evaluación - e Avaliação Psicológica, 32(2), 187-204. Recuperado de https://www.aidep.org/sites/default/files/201812/r32art9.pdf

Menéndez, S., Hidalgo, M. V., Lorence, B., \& Pérez, J. (2016). Assessing the level of risk of families supported by Child and Family Protection Services: Practitioners and mothers as informants. Journal of Social Work, 16(5), 595-609.doi:10.1177/1468017315583174

Nunnally, J., \& Bernstein, I. (1994). Psychometric theory (3rd ed.). New York, NY: McGrawHill.

Nunes, C., Jiménez, L., Menéndez, S., AyalaNunes, L., \& Hidalgo, V. (2016). Psychometric properties of an adapted version of the parental sense of competence (PSOC) scale for Portuguese at-risk parents. Child \& Family Social Work, 21(4), 433-441. doi:10.1111/cfs.12159
Olson, D. (2011). FACES IV and the circumplex model: Validation study. Journal of Marital and Family Therapy, 37(1), 64-80. doi:10.1111/j.1752-0606.2009.00175.x

Olson D. H., \& Gorall, D. M. (2003). Circumplex model of marital and family systems. In F. Walsh (Ed.), Normal Family Processes (3 ${ }^{\text {rd }}$ ed., pp. 514-547). New York: Guilford.

Olson, D. H., Portner, J. \& Bell, R. Q. (1982). FACES II: Family Adaptability and Cohesion Evaluation Scales. EUA: University of Minnesota.

Olson, D. H., Portner, J., \& Lavee, Y. (1985). FACES III. EUA: University of Minnesota.

Olson, D. H., Sprenkle, D. H., \& Russell, C. S. (1979). Circumplex model of marital and family systems: I. Cohesion and adaptability dimensions, family types, and clinical applications. Family Process, 18(1), 3-28. doi:10.1111/j.1545-5300.1979.00003.x

Olson, D. H., Waldvogel, L., \& Schlieff, M. (2019). Circumplex model of marital and family systems: An update. Journal of Family Theory \& Review, 11(2), 199-211. doi:10.1111/jftr.12331

Patrão, I., Pimenta, F., Água, J., \& Leal, I. P. (2020). Validação do Family Assessement Device (FAD) numa amostra de jovens portugueses. In $13^{\circ}$ Congresso Nacional de Psicologia da Saúde-Actas (pp. 773-781). Lisboa: Edições ISPA.

Pereira, M. G., \& Teixeira, R. (2013). Portuguese Validation of FACES-IV in Adult Children Caregivers Facing Parental Cancer. Contemporary Family Therapy, 35(3), 478490. doi:10.1007/s10591-012-9216-4

Pérez, J., Nunes, L., Nunes, C., \& Hidalgo, V. (2012). Estrés parental, cohesión y adaptación familiar en familias con menores en riesgo psicosocial: Un estudio comparativo entre Andalucía Occidental y el Algarve. Libro de actas del XI Congreso internacional de infancia maltratada: Construyendo puentes entre investigación y práctica (pp. 740-745). Oviedo: FAPMI \& ASACI.

Polaino-Lorente, A., \& Martínez Cano, P. (1995). La validez factorial de la" family functioning style scale" en una muestra de población española. Cuadernos de Terapia Familiar, 1995, 15-30. 
https://repositorioinstitucional.ceu.es/bitstrea m/10637/1985/1/Validez_A_Polaino\%26P_M artinez_Cuad_Tep_Fam_1995.pdf

Portner, J. (1981). Parent/adolescent relationships: Interaction types and the Circumplex model. Unpublished doctoral thesis, University of Minnesota.

Rapsey, C. M., \& Rolston, C. J. (2020). Fostering the family, not just the child: Exploring the value of a residential family preservation programme from the perspectives of service users and staff. Children and Youth Services Review, 108, 104505.

doi:10.1016/j.childyouth.2019.104505

Rodrigo, M. J., Máiquez, M. L., Martín, J. C., \& Byrne, S. (2008). Preservación familiar: Un enfoque positivo para la intervención con familias. Madrid: Pirámide.

Rolim, L., Lopes, A., Rodrigues, C., \& Coelho, T. (2006). Aplicação da Escala de Avaliação da Coesão e da Adaptabilidade Familiares-III (FACES III) a uma amostra portuguesa de doentes com polineuropatia amiloidótica familiar, (pp. 429-436). In Actas do $6^{\circ}$ Congresso Nacional de Psicologia da Saúde. Lisboa: Edições ISPA.

Rosas, E. P., Clavelina, F. G., Trillo, M. T., Coria, A. I., \& Ibáñez, S. L. (2003). Validez de constructo del cuestionario FACES III en español (México). Atención Primaria, 31(10), 624-630. doi:10.1016/S0212-6567(03)76319-7

Santos, S. V. (2008). Forma reduzida do Parenting Stress Index (PSI): Estudo Preliminar. XIII Conferência Internacional Avaliação Formas e Contextos. Braga: Universidade do Minho.

Schmidt, V., Barreyro, J. P., \& Maglio, A. L. (2009). Escala de evaluación del funcionamiento familiar FACES III: ¿modelo de dos o tres factores? Escritos de Psicología, 3(2), 30-36. Recuperado de http://scielo.isciii.es/scielo.php?script=sci_artt ext\&pid=S1989-38092010000100004

Silva, M. I. M. C., \& Sequeira, J. O. (2015). Validação da FACES IV: O funcionamento da família em diferentes etapas do ciclo vital. Dissertação de Mestrado não publicada, ISMT.
Smilkstein, G. (1978). The family APGAR: A proposal for a family function test and its use by physicians. Journal of Family Practice, 6(6), 1231-1239.

Sousa, L., \& Ribeiro, C. (2005). Percepção das famílias multiproblemáticas pobres sobre as suas competências. Psicologia, 19(1-2), 169191. Recuperado de http://www.scielo.mec.pt/pdf/psi/v19n12/v19n1-2a08.pdf

Tabachnick, B. G., \& Fidell, L. S. (2019). Using multivariate statistics (7th ed.). Boston, MA: Pearson Education.

Timmerman, M. E., \& Lorenzo-Seva, U. (2011). Dimensionality assessment of ordered polytomous items with parallel analysis. Psychological Methods, 16(2), 209-220. doi:10.1037/a0023353

Vandeleur, C. L., Preisig, M., Fenton, B. T., \& Ferrero, F. (1999). Construct validity and internal reliability of a French version of FACES III in adolescents and adults. Swiss Journal of Psychology, 58(3), 161-9. doi:10.1024//1421-0185.58.3.161

Vielva, I., Pantoja, L., \& Abeijón, A. (2001). Las familias y sus adolescentes ante las drogas. Bilbao: Instituto Deusto de Drogodependencias, Universidad de Deusto.

Zegers, B., Larraín, M. E., Polaino-Lorente, A., Trapp, A., \& Diez, I. (2003). Validez y confiabilidad de la versión española de la escala de Cohesión y Adaptabilidad Familiar (CAF) de Olson, Russell \& Sprenkle para el diagnóstico del funcionamiento familiar en la población chilena. Revista Chilena de NeuroPsiquiatría, 41(1). doi:10.4067/s0717-92272003000100006 